\title{
Calculation of the energetic neutral atom flux from a 3D time-dependent model heliosphere
}

\author{
O. Sternal ${ }^{1}$, H. Fichtner ${ }^{2}$, and K. Scherer ${ }^{2}$ \\ ${ }^{1}$ Institut für Experimentelle und Angewandte Physik: Heliosphärische Astroteilchenphysik, Christian-Albrechts-Universität, \\ Leibnizstrasse 11, 24118 Kiel, Germany \\ e-mail: sternal@physik.uni-kiel.de \\ ${ }^{2}$ Lehrstuhl für Theoretische Physik IV: Theoretische Weltraum- und Astrophysik, Ruhr-Universität Bochum, 44780 Bochum, \\ Germany \\ e-mail: hf@tp4.ruhr-uni-bochum.de
}

Received 25 April 2007 / Accepted 18 September 2007

\begin{abstract}
With the Interstellar Boundary Explorer (IBEX) the measurement of energetic neutral atoms (ENAs) will enable a remote sensing of the heliospheric boundary region where the solar wind plasma flow meets the interstellar medium. Together with Voyager $1(\& 2)$ in (and at least near) the healiosheath there will be a very fortunate instrument configuration in space that will allow us to obtain valuable information about the large-scale structure and dynamics of the heliosphere. Following ongoing theoretical discussions we have computed all-sky maps of the differential flux of ENAs produced from charge exchange with solar wind protons in a three-dimensionally structured and time-varying heliosheath. We employ a three-dimensional, time-dependent hydrodynamic model heliosphere to compute the production of ENAs by a generalisation of previous two-dimensional approaches. We find three-dimensionally structured all-sky ENA flux maps that are time-varying due to the solar activity cycle. While the study adds to the complexity of the task to correctly interpret the forthcoming measurements to be made with IBEX, it underlines the potential of this mission to significantly increase our understanding of the structure and dynamics of the heliosphere.
\end{abstract}

Key words. atomic processes - Sun: solar wind - Sun: activity - ISM: atoms

\section{Introduction}

The study of the heliosphere has gained increased interest during recent years for at least two reasons.

On the one hand it has been recognized as a shield against external influences from the local interstellar medium (LISM) on the terrestrial environment (Scherer et al. 2001, 2002; Florinski et al. 2004; Frisch 2006), which have been termed "interstellarterrestrial relations", for a review see Scherer et al. (2007). Of special interest is the heliosheath, i.e. the boundary region between the undisturbed solar wind and the LISM. It contributes significantly to the shielding effect, particularly with respect to the modulation of galactic cosmic ray spectra (Florinski et al. 2003; Scherer \& Ferreira 2005; Langner et al. 2006a). These high-energy particles are able to influence the climate on Earth (Svensmark 1998, 2006b,a; Fichtner et al. 2006; Scherer et al. 2007).

On the other hand the continuing success of the two Voyager missions has drawn attention to the problem of large-scale heliosphere structure as documented by several conferences and topical volumes (Scherer et al. 2001; Florinski et al. 2004; Frisch 2006), especially after the entry of Voyager 1 into the heliosheath in December 2004. The first measurements of energetic particles beyond the termination shock (Stone et al. 2005; Decker et al. 2005) revealed that the intensity maximum of anomalous cosmic rays is - at least along Voyager's trajectory - not located at the shock but rather ahead of the spacecraft. This can be explained, in principle, by test particle as well as self-consistent hybrid models combining the kinetic transport equation of energetic particles with a heliosheath computed from two- or three-dimensional hydrodynamic models of the heliosphere (Ferreira \& Scherer 2006; Langner et al. 2006b). These authors have demonstrated that the intensity of anomalous cosmic rays can peak several AU beyond the termination shock as a consequence of an adiabatic heating of the particles due to a nonvanishing compression of the subsonic solar wind flow in the heliosheath. Despite the new insights the Voyager missions have provided and despite the data they will record during their final decade (limited by the onboard power-supply), the information will remain limited to local regions of the outer heliosphere.

In order to obtain a global view of the structure of the heliosheath and to clarify the interaction of the solar wind with the LISM, the Interstellar Boundary Explorer (IBEX) mission (McComas et al. 2004, 2006) will measure energetic neutral hydrogen atoms (ENAs) that are mainly produced in the heliosheath, see, e.g., Gruntman (1992), Hsieh et al. (1992), Gruntman (1997), Gruntman et al. (2001), Scherer \& Fahr (2003b), Fahr \& Scherer (2004a), and the recent review by Fahr et al. (2007). These hydrogen ENAs are produced by charge exchange processes between solar wind and pick-up protons and interstellar hydrogen mainly in the inner heliosheath, and therefore their fluxes are sensitive to the physical state of this region. With IBEX the measurements of ENAs from the outer heliosphere will be carried to an unprecedented level: the already existing data recorded by the SOHO (Hilchenbach et al. 1998) and the Mars Express spacecraft (Galli et al. 2006) were obtained with instruments not designed for that particular task and consequently the interpretation of their findings remains unclear so far. 
Following a theoretical approach suggested by Scherer \& Fahr (2003a), Scherer \& Fahr (2003b) and Fahr \& Scherer (2004b) who computed solar wind and pick-up proton-induced ENA fluxes on the basis of a two-dimensional time-dependent model, we compute all-sky ENA flux maps using the threedimensional time-dependent model developed by Borrmann (2005), see also Borrmann \& Fichtner (2005). This paper supplements the other two 3-D ENA simulations published by Sternal et al. (2005) and Heerikhuisen et al. (2007). While the former paper was a preliminary short presentation of principle findings and the latter concentrated on ENA energies up to $200 \mathrm{~V}$, we extend the analysis here to higher energies, longer periods, and include discussions of both the implications of the Voyager measurements in the heliosheath (Decker et al. 2005) and recently revised charge exchange cross sections (Lindsay \& Stebbings 2005) for the ENA fluxes and the all-sky maps to be established with IBEX.

\section{The model}

Our computation of the production rates and resulting fluxes of hydrogen ENAs is based on the model by Scherer \& Fahr (2003a), Scherer \& Fahr (2003b) and Fahr \& Scherer (2004b). In the following, we describe the generalisation of their twodimensional approach in order to employ it for the threedimensional case. Note that we do not consider a contribution from charge exchange with pick-up protons in this paper.

\subsection{The model heliosphere}

The computations described and discussed in the following sections are based on a three-dimensional model of the dynamics of the heliosphere developed by Borrmann (2005), see also Borrmann \& Fichtner (2005). This model is formulated for the solar wind and the interstellar plasma as one fluid and the interstellar neutral gas as a second; any effects of self-consistently included galactic cosmic rays (see Chap. 10 in Scherer et al. 2007) are neglected. While the plasma is a quasi-neutral electronproton mixture, the neutral component is treated as a pure atomic hydrogen gas. The model adopts the often made assumption that magnetic fields do not have a direct dynamical influence (see, e.g., Fahr et al. 2000; Zank \& Müller 2003) and thus allow for a hydrodynamic treatment that is chosen for the neutral atom population.

We use the standard "reference model" that was defined by Borrmann \& Fichtner (2005) and is characterized by a solar wind at 1 AU with the "typical" values (see, e.g., Zank 1999) of $u_{\mathrm{SW}}=400 \mathrm{~km} \mathrm{~s}^{-1}$, a proton number density of $n_{\mathrm{p}}=8.3 \mathrm{~cm}^{-3}$ and a temperature of $T_{\mathrm{p}}=5 \times 10^{4} \mathrm{~K}$. The 11 -year variations of the solar wind speed are assumed to be mass flux conserving and described by

$\boldsymbol{u}_{\mathrm{SW}}(\vartheta, t)=\left[u(t)-\left(u(t)-u_{\min }\right) \Psi(\vartheta)\right] \boldsymbol{e}_{r}$

with the auxiliary functions

$u(t)=\left[u_{\max }-\left(u_{\max }-u_{\min }\right)\right] f(t)$

$f(t)=a+b \cos (\omega t) \exp [\cos (\omega t)]$

$\Psi(\vartheta)=1-\left(1+\exp \left[ \pm 2 \lambda_{1}\left(\vartheta-\lambda_{2}\right)\right]\right)^{-1}$

and the parameters $b=[\exp (1)+\exp (-1)]^{-1}, a=b \exp (-1)$ and $\omega=2 \pi /(11$ years) following Scherer $\&$ Fichtner (2004). With $\lambda_{1}=8$ and $\lambda_{2}=\pi / 2 \mp \pi / 6$ one obtaines the same velocity profile as assumed by, e.g., Hattingh (1998) or Ferreira et al. (2001), i.e.

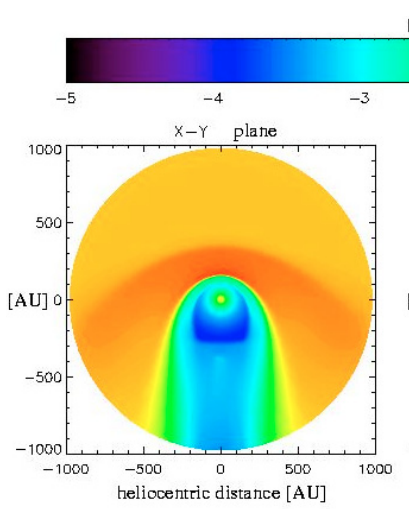

$\log (n)$
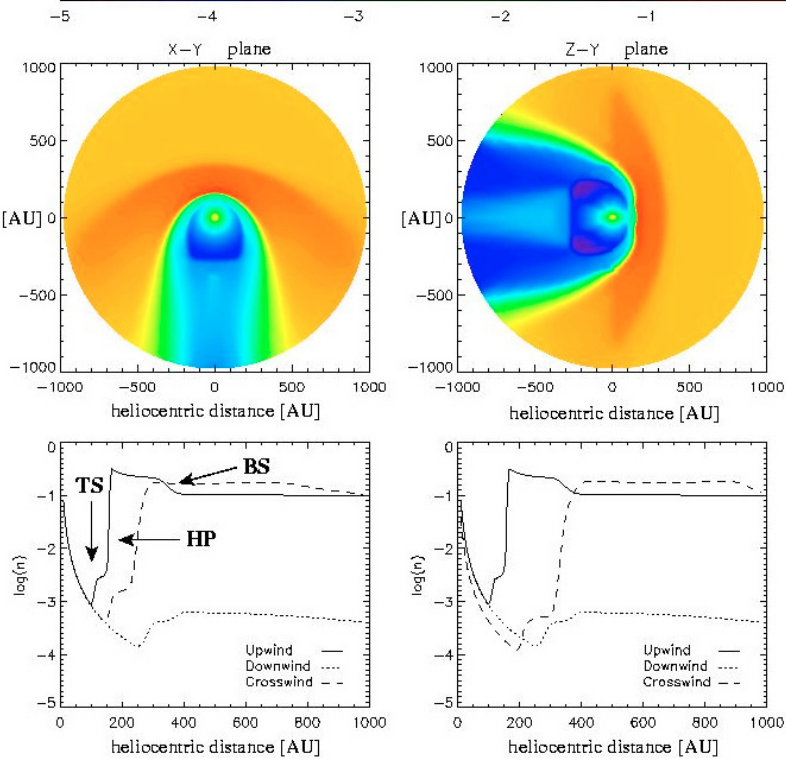

Fig. 1. Contour plots of the proton number density in the equatorial $(X-Y)$ and the meridional $(Y-Z)$ plane and the associated number density profiles in the upwind (solid line), downwind (dotted line) and the ecliptic crosswind (dashed line, left panels), and the polar (dashed line, right panels) direction in the heliosphere close to solar minimum. The termination shock, the heliopause and the bow shock are labeled TS, $\mathrm{HP}$ and BS, respectively.

a transition from slow to fast solar wind at $\pm 35^{\circ}$ latitude. Further we used $u_{\min }=400 \mathrm{~km} \mathrm{~s}^{-1}$ and $u_{\max }=800 \mathrm{~km} \mathrm{~s}^{-1}$. A typical result of the model is shown in Fig. 1.

At the outer boundary the LISM is assumed to be a homogeneous constant flow into the upwind heliosphere with a speed of $u_{\text {LISM }}=26 \mathrm{~km} \mathrm{~s}^{-1}$, a plasma density of $n_{\mathrm{p}, \mathrm{LISM}}=0.1 \mathrm{~cm}^{-3}$ and a neutral atom density of $n_{\mathrm{H}}=0.1 \mathrm{~cm}^{-3}$. Both components are assumed to have a temperature of $T_{\mathrm{p}, \mathrm{LISM}}=T_{\mathrm{H}}=8000 \mathrm{~K}$.

\subsection{The production of energetic neutral atoms}

Hydrogen ENAs are produced from charge exchange of solar wind and pick-up protons with interstellar neutral hydrogen atoms. Excluding the contribution from the latter, the corresponding differential production rate $\Psi_{\mathrm{ENA}, \mathrm{p}}$ is given by

$\Psi_{\mathrm{ENA}, \mathrm{p}}\left(\boldsymbol{r}, v_{\mathrm{ENA}}, \tau\right)=\left.\left(n_{\mathrm{p}} f_{\mathrm{p}}\left(v_{\mathrm{p}}\right) n_{\mathrm{H}} \sigma_{\mathrm{ex}}\left(v_{\text {rel }}\right) v_{\text {rel }}\right)\right|_{\boldsymbol{r}, \tau}$

with the production location and time $\boldsymbol{r}$ and $\tau$, the resulting speed of an ENA $v_{\text {ENA }}$, the local proton and hydrogen number densities $n_{\mathrm{p}}$ and $n_{\mathrm{H}}$, and the proton distribution function $f_{\mathrm{p}}$ in the solar wind rest frame

$f_{\mathrm{p}}\left(v_{\mathrm{p}}\right)=4 \pi\left(\frac{m_{\mathrm{p}}}{2 \pi k_{\mathrm{B}} T_{\mathrm{p}}}\right)^{\frac{3}{2}} \exp \left(-\frac{m_{\mathrm{p}} v_{\mathrm{p}}^{2}}{2 k_{\mathrm{B}} T_{\mathrm{p}}}\right)$.

Assuming that the hydrogen atoms are also characterized by a Maxwellian distribution function, the mean relative velocity $v_{\text {rel }}$ of a proton and a hydrogen atom is (Ripken \& Fahr 1983)

$v_{\text {rel }}=\sqrt{\frac{2 k_{\mathrm{B}} T_{\mathrm{H}}}{m}}\left(\frac{1}{\sqrt{\pi}} \exp \left(-\omega_{\mathrm{H}}^{2}\right)+\omega_{\mathrm{H}}\left(1+\frac{1}{2 \omega_{\mathrm{H}}}\right) \operatorname{erf}\left(\omega_{\mathrm{H}}\right)\right)$ 
with the auxiliary quantity

$\omega_{\mathrm{H}}=\sqrt{\frac{m}{2 k_{\mathrm{B}} T_{\mathrm{H}}}} \sqrt{v_{\mathrm{ENA}}^{2}+u_{\mathrm{H}}^{2}-2 v_{\mathrm{ENA}} u_{\mathrm{H}} \cos \Varangle\left(\boldsymbol{v}_{\mathrm{ENA}}, \boldsymbol{u}_{\mathrm{H}}\right)}$

and the error function

$\operatorname{erf}\left(\omega_{\mathrm{H}}\right)=\frac{2}{\sqrt{\pi}} \int_{0}^{\omega_{\mathrm{H}}} \exp \left(-z^{2}\right) \mathrm{d} z$.

For the charge exchange cross section $\sigma_{\text {ex }}$ we use the most recent result presented by Lindsay \& Stebbings (2005):

$\sigma_{\text {ex }, \mathrm{LS}}\left(v_{\mathrm{rel}}\right)\left[\mathrm{cm}^{2}\right]=\left(4.15-0.531 \ln \left(E_{\mathrm{rel}}\right)\right)^{2}$

$$
\cdot\left(1-\exp \left(-\frac{67.3}{E_{\text {rel }}}\right)\right)^{4.5}
$$

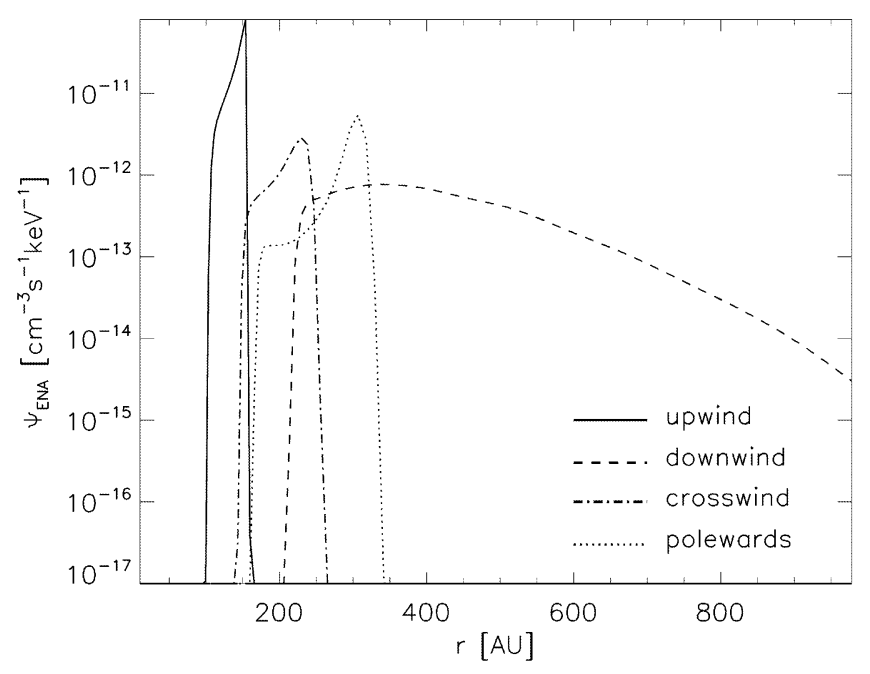

where the projectile energy $E_{\text {rel }}=m_{\mathrm{p}} v_{\text {rel }}^{2} / 2$ has to be inserted in units of $\mathrm{keV}$. While this representation appears to be the optimum consensus in view of various suggestions (see the discussion in Fahr et al. 2007), an uncertainty remains, see the discussion in Sect. 2.3.3 below.

Note that the solar wind parameters vary in time due to solar activity and that in turn also the ENA production rate is not constant. Typical production rates of hydrogen ENAs with a kinetic energy of $0.1 \mathrm{keV}$ are shown in Fig. 2. These results are valid for an ENA production from solar wind protons, i.e. do not include any contribution from charge exchange with pick-up protons.

Obviously, ENAs are significantly produced only in the (inner) heliosheath between the termination shock (TS) and the heliopause (HP). The curves that represent the lines-of-sight along the upwind, the downwind, the polar and the crosswind directions nicely reflect the fact that the heliosheath is relatively narrow in the upwind, the polar and the crosswind directions, but extends far in the downwind direction, where it is referred to as the heliotail. Comparing both solar activity phases, one can find small differences in the production rates in the polar regions. These results clearly demonstrate that the dominant solar wind contribution to the ENA production comes from the shocked and heated subsonic solar wind beyond the termination shock. Consequently, this is the region of the heliosphere that can be probed best with ENA measurements.

\subsection{The flux of energetic neutral atoms}

After the charge exchange reaction an ENA can move in any direction and thus - because it is not influenced by the heliospheric magnetic field - contribute to a measurable ENA flux near the Earth (e.g. Gruntman 1992; Hsieh et al. 1992; Gruntman 1997), see the sketch shown in Fig. 3.

The differential ENA flux can be calculated by a line-of-sight integration of the production rate:

$$
\begin{aligned}
\Phi_{\mathrm{ENA}, \mathrm{p}}\left(v_{\vartheta, \varphi}, \vartheta, \varphi, t\right) & =\frac{1}{4 \pi} \int_{\mathrm{IB}}^{\mathrm{OB}} \Psi_{\mathrm{ENA}, \mathrm{p}}\left(\boldsymbol{r}, v_{\mathrm{ENA}}, \tau\right) \mathrm{d} s \\
& =\left.\frac{1}{4 \pi} \int_{\mathrm{IB}}^{\mathrm{OB}}\left(n_{\mathrm{p}} f_{\mathrm{p}}\left(v_{\mathrm{p}}\right) n_{\mathrm{H}} \sigma_{\mathrm{ex}}\left(v_{\mathrm{rel}}\right) v_{\mathrm{rel}}\right)\right|_{\boldsymbol{r}, \tau} \mathrm{d} s .
\end{aligned}
$$

The two angles $(\vartheta, \varphi)$ denote the heliographic latitude and longitude of the chosen line-of-sight, while $v_{\vartheta, \varphi}$ is the ENA velocity in the solar rest frame:

$v_{\vartheta, \varphi}=\sqrt{\frac{2 E_{\mathrm{ENA}}}{m_{\mathrm{H}}}}=\left|\boldsymbol{u}_{\mathrm{SW}}+\boldsymbol{v}_{\mathrm{p}}\right|$.

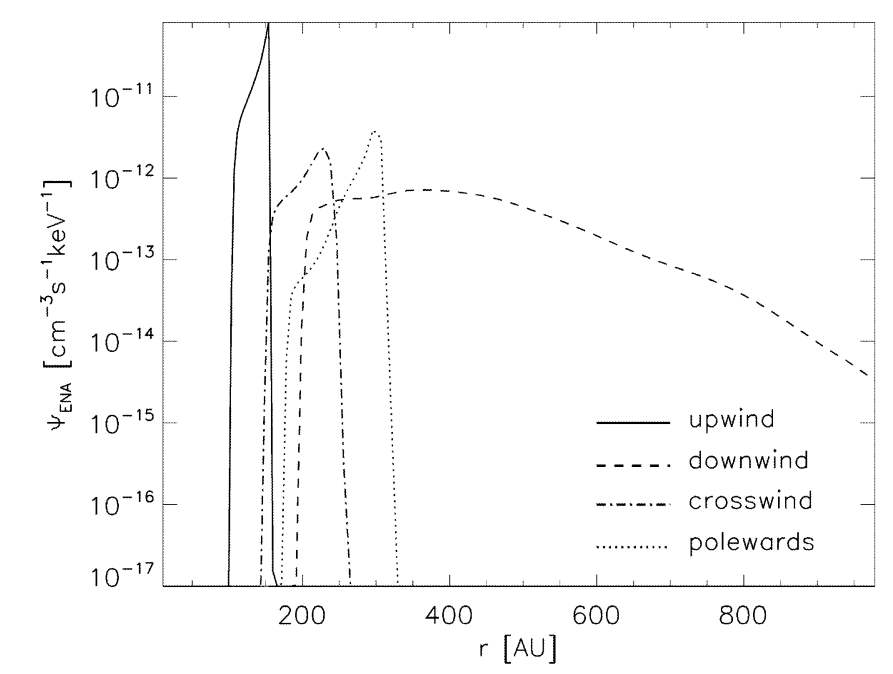

Fig. 2. Differential proton-induced ENA production rate for $E_{\mathrm{ENA}}=$ $0.1 \mathrm{keV}$ during solar maximum (upper panel) and solar minimum (lower panel) conditions for four different lines-of-sight, namely along the upwind, the downwind, the polar and the crosswind directions.

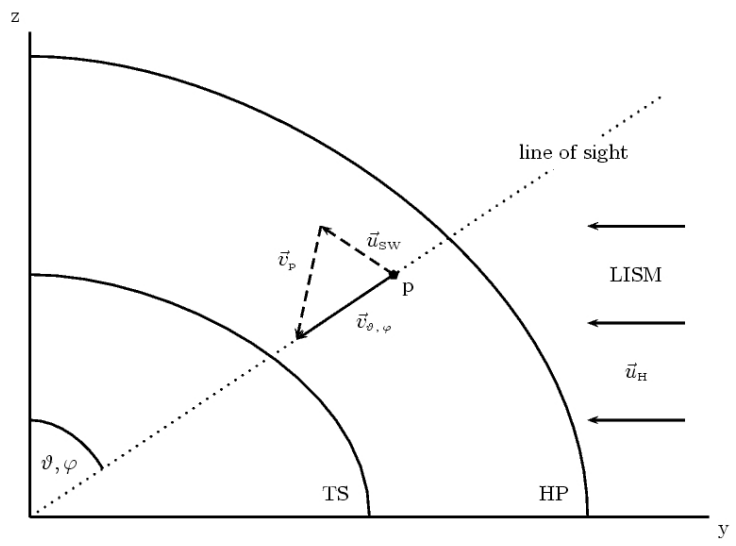

Fig. 3. The velocity $\boldsymbol{v}_{\vartheta, \varphi}$ of a single solar wind proton (p) in the observer rest frame is composed of the solar wind convection velocity $\boldsymbol{u}_{\mathrm{SW}}$ and the particle's thermal velocity $\boldsymbol{v}_{\mathrm{p}}$. So after the charge exchange reaction in the heliosheath between the termination shock (TS) and the heliopause (HP) the new ENA moves with $\boldsymbol{v}_{\mathrm{ENA}}=\boldsymbol{v}_{\vartheta, \varphi}=\boldsymbol{u}_{\mathrm{SW}}+\boldsymbol{v}_{\mathrm{p}}$. If this velocity vector points palong a line-of-sight, the ENA can contribute to a corresponding flux near Earth and, therefore, be detected with a kinetic energy of $E_{\mathrm{ENA}}=\frac{1}{2} m_{\mathrm{H}} v_{\vartheta, \varphi}^{2}$. The two angles $(\vartheta, \varphi)$ are the heliographic latitude and longitude of the chosen line-of-sight. 

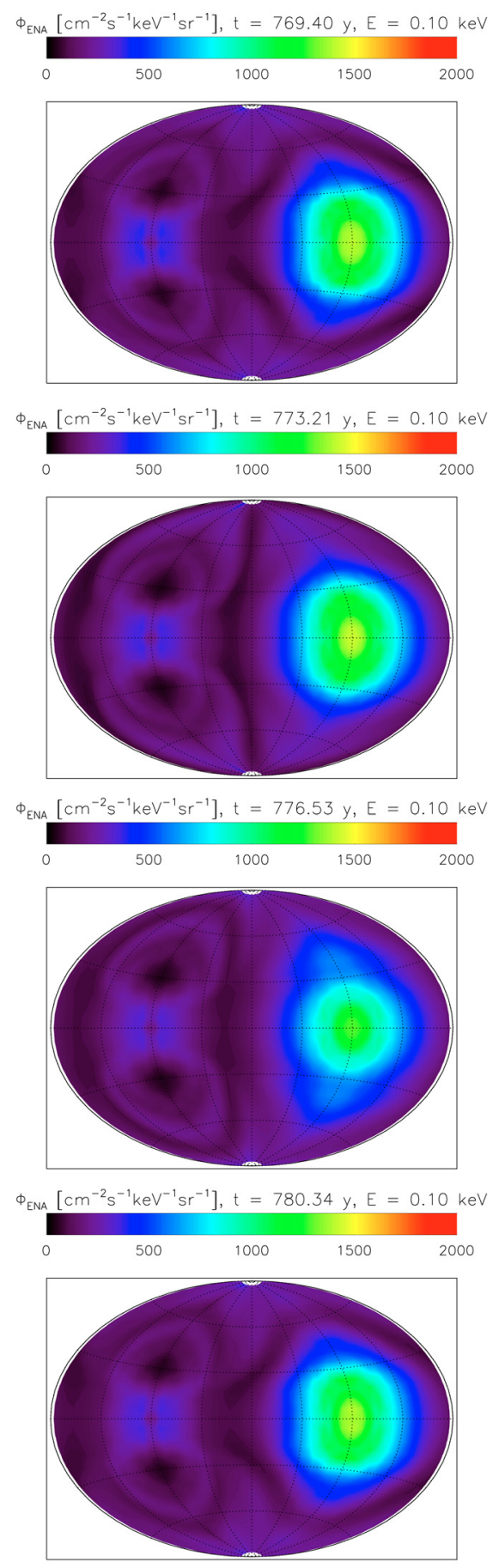

Fig. 4. All-sky maps of the solar wind proton-induced ENA flux for $E_{\mathrm{ENA}}=0.1 \mathrm{keV}$ in an Aitoff-projection for four points in time during an 11-year solar activity cycle beginning with solar maximum. The snapshots are separated by about four years. At this comparatively low energy, the almost axisymmetric flux shows only minor variations with time concentrated at higher latitudes as a result of the change from slow to fast solar wind. Note that ionization losses of the ENAs are neglected.

In order to obtain the correct flux the inner (IB) and outer (OB) integration boundaries have to be set such that the ENA production occurs in the distance range between them. Caution must be exercised regarding the production and detection time of an ENA. For the computations the detection time $t$ of an ENA is defined by the production time plus the time required for an ENA to move from the production location to the detector:

$t=\tau+\frac{s(\vartheta, \varphi)}{v_{\vartheta, \varphi}}$

where $s$ denotes the distance between production and detection location. In the following we calculate the differential ENA fluxes at 0.1 and $1 \mathrm{keV}$ near the Earth orbit on the basis of the three-dimensional time-dependent model described in Sect. 2.1.

\subsubsection{The differential ENA flux at $0.1 \mathrm{keV}$}

The differential flux of $0.1 \mathrm{keV}$ ENAs in the reference heliosphere described in Borrmann \& Fichtner (2005) that is regularly "breathing" with an 11-year period due to solar activity variations of the solar wind parameters is given in Fig. 4.

The four maps have a very similar, almost axisymmetric structure. Only at higher latitudes, where the strongest change in the solar wind velocity occurs, a very weak variation during the solar activity cycle is identified. At this energy the basic structure of the maps is characterized by two maxima: there is a strong maximum in the upwind direction caused by the high ENA production rate in a narrow heliosheath, compare with the solid line in Fig. 2. A weak secondary maximum is located in the heliotail where the production rate is much lower, compare with the dashed line in Fig. 2, but extends over several hundred AU, thus partly compensating for the lower rates. These findings correspond to those presented by Heerikhuisen et al. (2007), see especially their results for $200 \mathrm{eV}$ (right panel in their Fig. 1b). The flux levels computed by these authors based on the study by Heerikhuisen et al. (2006) are of the same order, even though they (i) employed a kinetic modelling for the neutral atoms resulting in velocity distributions broader than the Maxwellian used here for the computation of the relative speed according to Eq. (4), (ii) a different charge exchange cross section, and (iii) a higher number density of the interstellar gas.

\subsubsection{The differential ENA flux at $1.0 \mathrm{keV}$}

This higher energy, which is in the prime measurement interval of IBEX, has not been considered by Heerikhuisen et al. (2007). Our results, again for the time-varying reference heliosphere, are displayed in Fig. 5.

There are four major differences between the flux at 1 and at $0.1 \mathrm{keV}$. First, the $1 \mathrm{keV}$ flux levels are lower by about two orders of magnitude. This is expected from the previous modelling (e.g. Fahr \& Scherer 2004a) and reflects a negative slope of the ENA energy spectrum in this range, see also Gruntman et al. (2001). Second, the axisymmetry seen at the low-energy fluxes is broken due to the stronger effect of the solar activityinduced variations of the solar wind. Third, the maximum ENA flux comes from polar and/or high-latitude regions. And, fourth, the clearly visible time dependence is revealed by a shift of the ENA flux maximum from the upwind to the downwind hemisphere and back during a solar cycle.

The general differential flux level at $1 \mathrm{keV}$ of less than 8 particles $/ \mathrm{cm}^{2} / \mathrm{s} / \mathrm{sr} / \mathrm{keV}$ is consistent with the estimates by Gruntman et al. (2001), compare their Fig. 7. As mentioned above, because for the present analysis Maxwellian distributions for the solar wind protons are used and any contribution from pick-up ions is neglected, the resulting ENA fluxes should be considered as lower limits. The non-axisymmetric, threedimensional structure of the all-sky maps at this energy is an interesting new feature, because if the highest flux and the clearest time variations are concentrated at high latitudes as shown 

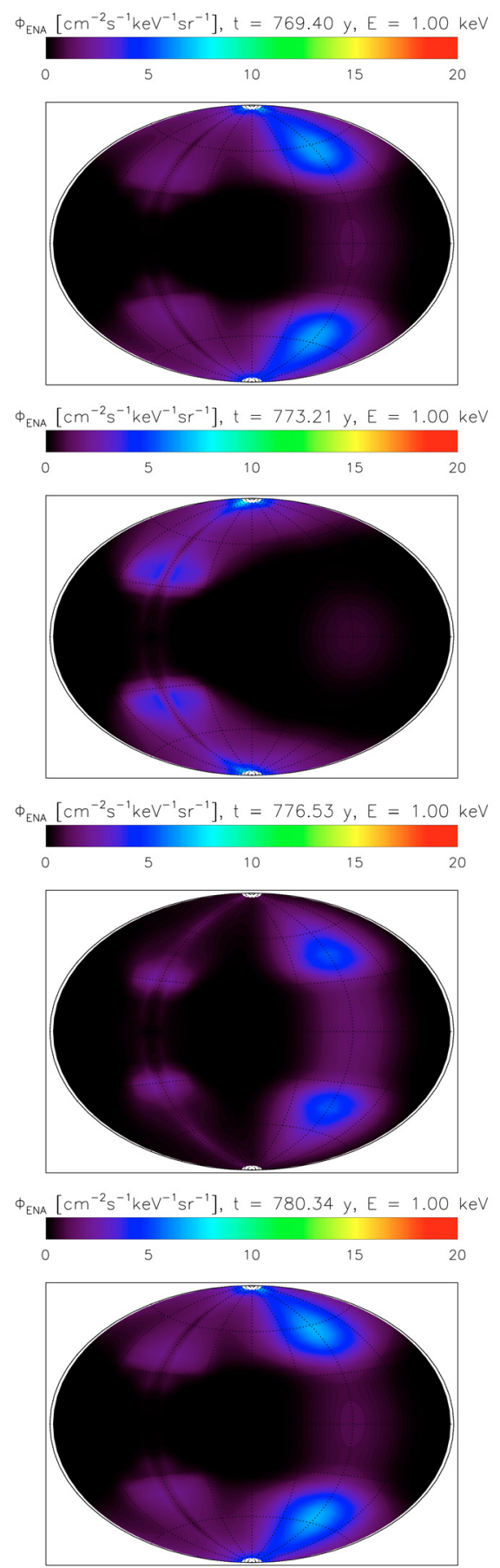

Fig. 5. Same as Fig. 4 but for $1.0 \mathrm{keV}$. The computed particle flux at this energy is about two orders of magnitude lower than that at at $E_{\mathrm{ENA}}=0.1 \mathrm{keV}$. It is concentrated in the polar regions and shows a strong variability with time. The maximum flux is shifted from the upwind- to the downwind-hemisphere during the solar cycle. Note that ionization losses of ENAs on their way to the detector are neglected.

in Fig. 5. It is most easily accessible through measurements with the IBEX spacecraft as the polar regions are regularly scanned in the shortest possible time intervals given by the $1 \mathrm{rpm}$ spin rate (see McComas et al. 2004; and McComas et al. 2006).

\subsubsection{Parameter dependence of the differential ENA fluxes}

The differential ENA fluxes are sensitive to various parameters. Rather than being simply linear, the influence of the inner and outer boundary conditions is nonlinear because not only the ENA production but the large-scale structure of the heliosphere depends on the charge exchange coupling of plasma and neutral gas. Because of the central role played by the charge exchange process, we have studied the consequences of the known uncertainties regarding the cross sections.

As reviewed in Fahr et al. (2007), an update of the energy dependence of the proton-hydrogen charge exchange cross section has been presented by Lindsay \& Stebbings (2005). The latter authors derived a "consensus" fit to the available data in the energy range from $5 \mathrm{eV}$ to $200 \mathrm{keV}$ given in Eq. (7).

Therefore we compare an $0.1 \mathrm{keV}$ and an $1 \mathrm{keV}$ ENA flux map computed with one of the two traditionally used cross sections, that suggested by Fite et al. (1962)

$\sigma_{\text {ex,Fi }}\left(v_{\text {rel }}\right)=\left(2.1 \times 10^{-7}-9.2 \times 10^{-9} \ln \left(v_{\text {rel }}\right)\right)^{2}$

and with $\sigma_{\text {ex,LS }}$ in Fig. 6 . The maps show the expected difference: the overestimation of the differential ENA fluxes computed with $\sigma_{\text {ex,Fi }}$ increases with energy and mounts to $30 \%$.

Another interesting parameter that has a direct influence on the ENA fluxes from the heliosheath is the solar wind speed in that region. The first measurements made by the Voyager 1 spacecraft in this region point to a solar wind speed of below $50 \mathrm{~km} \mathrm{~s}^{-1}$, i.e. a value significantly below the originally expected range of 100-200 $\mathrm{km} \mathrm{s}^{-1}$ (depending on the compression ratio of the termination shock). The indirectly observed low value has, so far, not been reproduced with simulations of the large-scale heliosphere. We therefore studied the resulting effect by computing a $0.1 \mathrm{keV}$ and a $1 \mathrm{keV}$ ENA flux map for a solar wind velocity that is (artificially) reduced by $50 \%$ in the heliosheath. Our findings are displayed in Fig. 7. The results reveal that such reduction can have a rather strong effect amounting to a flux increase of up to a factor of two and three at $0.1 \mathrm{keV}$ and $1 \mathrm{keV}$, respectively. While more detailed investigations should be performed as soon as such low velocities are observationally confirmed (e.g., with the Voyager 2 spacecraft whose plasma instrumenst are still functioning) and can be self-consistently simulated with models of the large-scale structure of the heliosphere, the potential significance of this parameter is obvious from our findings.

Finally, there is the problem of ENA losses due to ionisation on their way from the outer heliosphere to 1 AU. Gruntman (1992), see also Gruntman et al. (2001), estimated these losses to be (40-50)\% at $0.1 \mathrm{keV}$ with a gradual decrease to about $10 \%$ at $6 \mathrm{keV}$. These estimates are in qualitative agreement with those in Fahr \& Scherer (2004b), although made there in a somewhat different context. The computed maps shown above are not yet corrected for such ENA losses, so that the actual fluxes at 0.1 and $1 \mathrm{keV}$ will be lower by about $50 \%$ and $15 \%$, respectively. While this will not affect the computed time dependences, which are the central result of the present paper, a more detailed and quantitative calculation of ENA losses will be the subject of forthcoming work.

\section{Conclusions}

The first computations of the differential flux of energetic neutral atoms produced in the (inner) heliosheath of a threedimensionally structured and time-varying heliosphere revealed two main characteristics of the corresponding all-sky maps. First, the nearly perfect axisymmetry of the differential flux distribution that can be seen at low energies (e.g., $0.1 \mathrm{keV}$ ) vanishes towards higher energies. Second, due to the solar activity cycle, 

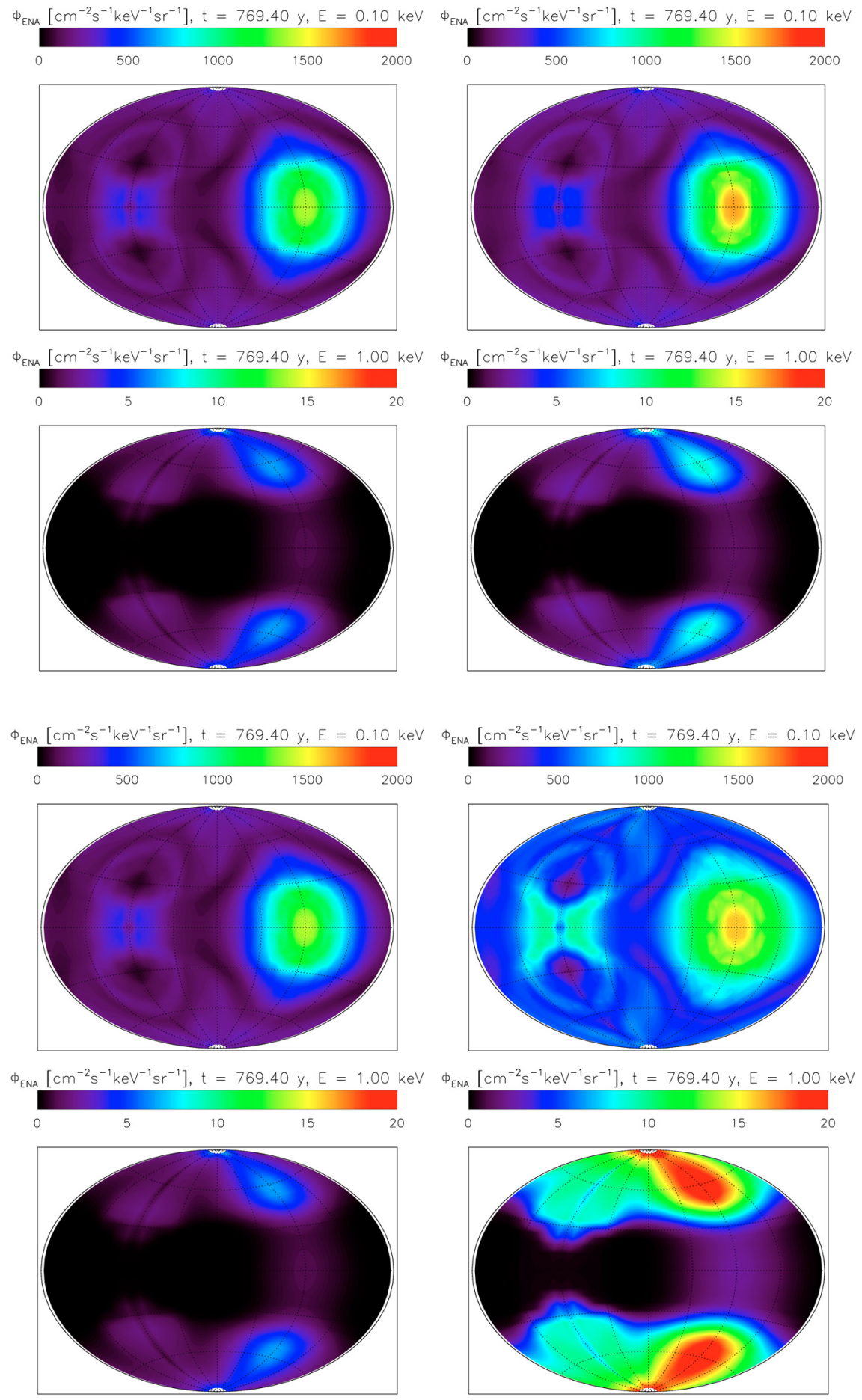

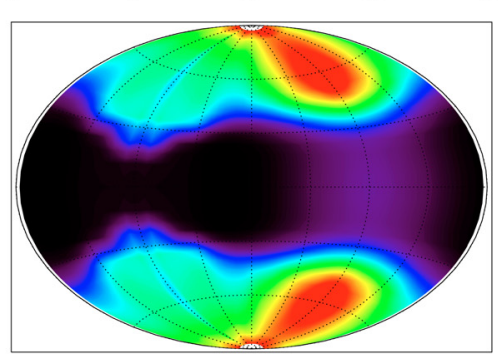

Fig. 6. The left two panels display the ENA flux data discussed before, i.e. those computed with $\sigma_{\text {ex,LS }}$ in Eq. (7), while the all-sky-maps on the right show the results obtained with $\sigma_{\text {ex,Fi }}$ in Eq. (11).
Fig. 7. The left two panels display the ENA flux data discussed before, i.e. those computed with $\sigma_{\text {ex,LS }}$ in Eq. (7), while the all-sky-maps on the right show the results obtained with a solar wind speed reduction by $50 \%$ in the heliosheath. Note that colour codings were fit to the higher fluxes in the right panels. there is a pronounced time dependence of the differential ENA fluxes at higher energies (here $1 \mathrm{keV}$ ) that is concentrated at the polar regions, where the regular changes between fast and slow solar wind are strongest. If confirmed with future modelling, this time variation should be easily detectable via measurements with the IBEX spacecraft as the polar regions are regularly scanned in the shortest possible time intervals given by the spin rate of $1 \mathrm{rpm}$ (see McComas et al. 2004; and McComas et al. 2006).

These findings have been supplemented with a study of two critical quantities, namely the proton-hydrogen charge exchange cross section and the solar wind velocity in the heliosheath where the overwhelmingly dominant contribution of the ENA flux is produced. We found that both parameters do have a significant influence. Comparing the most recent consensus fit to laboratory data (Lindsay \& Stebbings 2005) with the traditionally employed cross sections provided by Fite et al. (1962) revealed flux difference from up to $30 \%$ below $1 \mathrm{keV}$ and more above. A $50 \%$ reduction of the solar wind velocity in the heliosheath results in even higher flux changes by up to a factor of three.

Our results add to the complexity of the forthcoming task to interpret the all-sky ENA flux maps to be recorded with IBEX. Beyond the fact that these maps will not provide snapshots of the state of the outer heliosphere, because they will be recorded over periods of several months and because the propagation times of the solar wind plasma to different locations in the heliosheath 
and of the ENAs back to the inner heliosphere results in a memory effect (Scherer \& Fahr 2003b), and, therefore, will have an integration aspect, they are expected to exhibit a characteristic time dependence. While, consequently, the correct interpretation of these maps will be a challenge to future modelling, it will significantly increase our understanding of the large-scale structure and dynamics of the heliosphere.

Acknowledgements. We thank the referee for constructive comments and pointing out a missing normalization factor.

\section{References}

Borrmann, T. 2005, Ph.D. Thesis, Ruhr-Universität Bochum, Germany Borrmann, T., \& Fichtner, H. 2005, Adv. Space Res., 35, 2091

Decker, R. B., Krimigis, S. M., Roelof, E. C., et al. 2005, Science, 309, 2020

Fahr, H.-J., \& Scherer, K. 2004a, Astrophys. Space Sci. Trans., 1, 3

Fahr, H.-J., \& Scherer, K. 2004b, Astrophys. Space Sci. Trans., 1, 3

Fahr, H. J., Kausch, T., \& Scherer, H. 2000, A\&A, 357, 268

Fahr, H.-J., Fichtner, H., \& Scherer, K. 2007, Rev. Geophys., in press

Ferreira, S. E. S., \& Scherer, K. 2006, ApJ, 642, 1256

Ferreira, S. E. S., Potgieter, M. S., Burger, R. A., Heber, B., \& Fichtner, H. 2001, J. Geophys. Res., 106, 24979

Fichtner, H., Scherer, K., \& Heber, B. 2006, Atm. Chem. Phys. Disc., 6, 10811

Fite, W. L., Smith, A. C. H., \& Stebbings, R. F. 1962, Proc. Roy. Soc. A, 286, 527

Florinski, V., Zank, G. P., \& Pogorelov, N. V. 2003, J. Geophys. Res. (Space Phys.), 1

Florinski, V., Zank, G. P., Jokipii, J. R., Stone, E. C., \& Cummings, A. C. 2004, ApJ, 610, 1169

Frisch, P. C. 2006, Solar Journey: The Significance of our Galactic Environment for the Heliosphere and Earth, ed. P. C. Frisch, University of Chicago, IL, USA, Astrophysics and Space Science Library, 338 (Springer Dordrecht)

Galli, A., Wurz, P., Barabash, S., et al. 2006, ApJ, 644, 1317

Gruntman, M. A. 1992, Planet. Space Sci., 40, 439

Gruntman, M. 1997, Rev. Scientific Instrum., 68, 3617
Gruntman, M., Roelof, E. C., Mitchell, D. G., et al. 2001, J. Geophys. Res., 106, 15767

Hattingh, M. 1998, Ph.D. Thesis, Potchefstroomse Universiteit vir Christelike Hoër Onderwys, RSA

Heerikhuisen, J., Florinski, V., \& Zank, G. P. 2006, J. Geophys. Res. (Space Phys.), 111, 6110

Heerikhuisen, J., Pogorelov, N. V., Zank, G. P., \& Florinski, V. 2007, ApJ, 655, L53

Hilchenbach, M., Hsieh, K. C., Hovestadt, D., et al. 1998, ApJ, 503, 916

Hsieh, K. C., Shih, K. L., Jokipii, J. R., \& Grzedzielski, S. 1992, ApJ, 393, 756

Langner, U. W., Potgieter, M. S., Fichtner, H., \& Borrmann, T. 2006a, ApJ, 640, 1119

Langner, U. W., Potgieter, M. S., Fichtner, H., \& Borrmann, T. 2006b, J. Geophys. Res. (Space Phys.), 111, A01106

Lindsay, B. G., \& Stebbings, R. F. 2005, J. Geophys. Res. (Space Phys.), 110, 12213

McComas, D., Allegrini, F., Bochsler, P., et al. 2004, in Physics of the Outer Heliosphere, AIP Conf. Proc., 719, 162

McComas, D. J., Allegrini, F., Bartolone, L., et al. 2006, in Physics of the Inner Heliosheath, ed. J. Heerikhuisen, V. Florinski, G. P. Zank, \& N. V. Pogorelov, AIP Conf. Proc., 858, 241

Ripken, H. W., \& Fahr, H. J. 1983, A\&A, 122, 181

Scherer, K., \& Fahr, H. J. 2003a, Annales Geophysicae, 21, 1303

Scherer, K., \& Fahr, H.-J. 2003b, A\&A, 404, L47

Scherer, K., \& Ferreira, S. E. S. 2005, A\&A, 442, L11

Scherer, K., \& Fichtner, H. 2004, A\&A, 413, L11

Scherer, K., Fichtner, H., Borrmann, T., et al. 2007, Space Sci. Rev., 65

Scherer, K., Fichtner, H., Fahr, H. J., \& Marsch, E. 2001, The Outer Heliosphere: The Next Frontiers

Scherer, K., Fichtner, H., \& Stawicki, O. 2002, J. Atmospheric Terrestrial Phys., 64, 795

Sternal, O., Scherer, K., \& Fichtner, H. 2005, in Solar Wind 11/SOHO 16, Connecting Sun and Heliosphere, ESA SP-592, 383

Stone, E. C., Cummings, A. C., McDonald, F. B., et al. 2005, Science, 309, 2017

Svensmark, H. 1998, Phys. Rev. Lett., 81, 5027

Svensmark, H. 2006a, Astron. Notes, 327, 871

Svensmark, H. 2006b, Astron. Notes, 327, 866

Zank, G. P. 1999, Space Sci. Rev., 89, 413

Zank, G. P., \& Müller, H.-R. 2003, J. Geophys. Res. (Space Phys.), 7 